\title{
El valor agregado de un sistema de gestión ambiental más allá de la certificación
}

\section{The added value of an environmental management system beyond certification}

\section{Javier Augusto Vera Solano .; Julio Eduardo Cañón Barriga}

Universidad de Antioquia. Grupo de investigación: GAIA

\section{Resumen}

Este documento analiza cómo un modelo de gestión bien adoptado puede convertirse en una herramienta que genera beneficios ambientales palpables cuando los programas que en él se implementan efectivamente cumplen con su objetivo y además se hace una valorización real de las salidas de los procesos como residuos sólidos, aguas residuales, consumo de energía y comportamientos ambientales de los trabajadores que se involucran en el propio funcionamiento del modelo de gestión ambiental.

La metodología utilizada hace parte de una revisión de literatura científica que se ha venido realizando acerca del desempeño ambiental de las empresas certificadas en ISO 14001 y de los variables internas y externas que pueden incidir en el mismo. Existe un alto porcentaje de empresas que tienden a integrar en su nivel organizacional el aspecto ambiental sea solo por mantener un reconocimiento o porque efectivamente desean contribuir a la solución de la reducción de la contaminación. Los resultados parciales indican que un gran número de empresas solo implementan los sistemas de gestión ambiental por un interés de mejora de imagen, competitividad del mercado, presiones externas entre otras y muy pocas adoptan cambios fundamentales en la parte ambiental, contrariando la premisa principal de la política ambiental que muchas organizaciones divulgan.

Palabras clave: desempeño, residuos, valoración

\section{Abstract}

This paper analyzes how a well-adopted management model can generate tangible environmental benefits when the programs implemented in it, effectively meet its objective and also make a real appreciation of the outputs of processes such as solid waste, wastewater, energy consumption and environmental behavior of workers who are involved in the operation of the environmental management model. The methodology used is part of a review of scientific literature about the environmental performance of companies certified in ISO 14001 and the internal and external variables that may affect them. There are a 


\section{7}

high percentage of companies that tend to integrate the environmental aspect at their organizational level, either just to maintain recognition or because they really want to contribute to the solution of the reduction of pollution issues.

The partial results indicate that a large number of companies only implement environmental management systems due to an image improvement interest, market competitiveness, and external pressures among others, whereas few adopt fundamental changes in the environmental aspects, contrary to the main premise of the environmental policy that many organizations report.

Keywords: assessment, performance, waste.

\section{INTRODUCCIÓN}

Actualmente en las empresas productivas domina la idea sobre las motivaciones económicas para la implantación de sistemas de gestión deben ir acompañadas de motivos de marketing. La certificación ISO 14001 a este respecto es ampliamente considerada la herramienta de elección para impulsar los esfuerzos de minimización de residuos (Singh, Brueckner, \& Kumar, 2015). Las empresas tienen procesos productivos que generan subproductos que no se pueden aprovechar y deben ser tratados. Para esto deben buscar un tipo de solución, pero se limitan por costos y prefieren gestionar la salida de los mismos.

No todos los residuos se pueden valorizar, pero si la empresa tiene adoptado un sistema de gestión ambiental, y los subproductos que quedan de las salidas de los procesos son aprovechables, la organización debe utilizar toda esa estructura del (SGA) para devolverlos al sector productivo, no como desecho sino como un producto que tiene un valor más allá de lo económico: un valor ambiental. Acá es donde entra el concepto de valor agregado de un producto, ya que de la gestión de los desechos por ejemplo se reduce el volumen de residuos que llega a vertederos. De hecho, según (Tamayo Orbegozo, Vicente Molina, \& Izaguirre Olaizola, 2012) una gestión eficiente de la variable medioambiental puede proporcionar numerosas oportunidades de negocio a la empresa, sustituyendo materias primas provenientes de recursos naturales por estos materiales recuperados.

\section{1. Sistema de gestión ambiental}

El SGA puede definirse como: "Un sistema estructurado de gestión, integrado con la actividad de gestión total de la organización, que incluye la estructura organizativa, la planificación de las actividades, las responsabilidades, las prácticas, los procedimientos, los procesos y los recursos para desarrollar, implantar, llevar a efecto, revisar y mantener al día los compromisos en materia de protección medioambiental que suscribe la organización" (Rey, 2008).

Los SGA son la herramienta con la cual la empresa determina las acciones o medidas a 
88

tomar cuando algunos de sus procesos, por las actividades implícitas en los mismos, generan impactos ambientales. Actualmente la adopción de estos sistemas se ha incrementado a nivel mundial, siendo el modelo ISO 14001 el más acogido (Escobar Cardenas, 2009)

\subsection{El Valor agregado en la parte ambiental}

El valor agregado es el valor adicional que obtienen los bienes y servicios al ser convertidos en el proceso productivo, es la característica extra que un bien o servicio ofrece con el propósito de generar mayor valor dentro de la percepción del consumidor.

Por ejemplo la mejor opción para optimizar la gestión de residuos sólidos es dar un valor agregado a los mismos mediante una recuperación y transformación de ellos. El valor agregado ambiental (VAA) pone en primer plano la inversión en la calidad ambiental, métodos y técnicas de producción que buscan los menores impactos en el entorno, el uso más eficiente de los insumos y la alta calidad de los productos por medio de condiciones rigurosas (Evia \& Gudynas, 2000).

\subsection{Las Certificaciones ambientales}

La certificación ambiental es una acreditación obtenida por determinados productos, servicios, procesos, o sistemas de gestión, que "acredita" que todos o determinados procesos correspondientes a los mismos, se han llevado a cabo de un modo respetuoso con el medio ambiente y conforme a la normativa ambiental respectiva (Galarza, 2012).Existen varios tipos de certificación ambiental (ver la figura 1 ), las cuales vienen siendo adoptados por las empresas de forma voluntaria con el propósito de mejorar su desempeño ambiental.

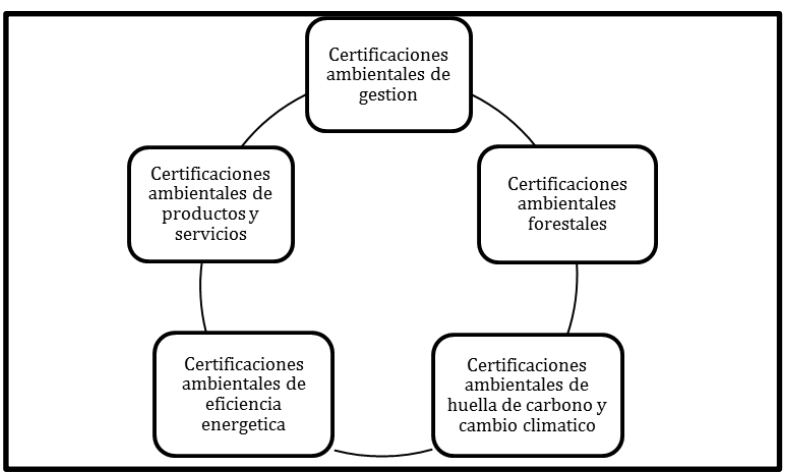

Figura 1 Tipo de certificaciones ambientales

\subsection{Valorización y aprovechamiento de los desechos industriales como valor agregado de los sistemas de gestión ambiental}

Un análisis del uso dado a los residuos obtenidos de distintos sectores agroindustriales ver Figura 2 demuestra que la industria del fique aprovecha el $2 \%$ de la biomasa producida, la cervecera emplea el $8 \%$ de los nutrientes del grano, mientras que la de aceite de palma y de celulosa menos del $9 \%$ y $30 \%$ de la biomasa producida, es empleada respectivamente. En la industria del café solo el $9.5 \%$ del peso del fruto fresco es aprovechado para la preparación de la bebida, quedando un $90.5 \%$ como residuo (Cury R, Aguas M, Martinez M, \& et 
al, 2017). Otro ejemplo de agroindustria de gran impacto en cuanto a la producción de residuos lo constituye la producción de papel, donde solo el $30 \%$ de la materia prima es aprovechada (Agrocadenas Ministerio de Agricultura y desarrollo rural, 2006), el resto de porcentaje es desperdiciado y dispuesto como desecho sin ningún tipo de valor. Es más, muchos de estos desechos por ejemplo la cascara de naranja, que es un residuo agrícola, abundante y de bajo costo están siendo estudiados ya que pueden reutilizarse $y$ postularse como un material bioadsorvente (Pinzon Bedoya \& Cardona Tamayo, 2008)

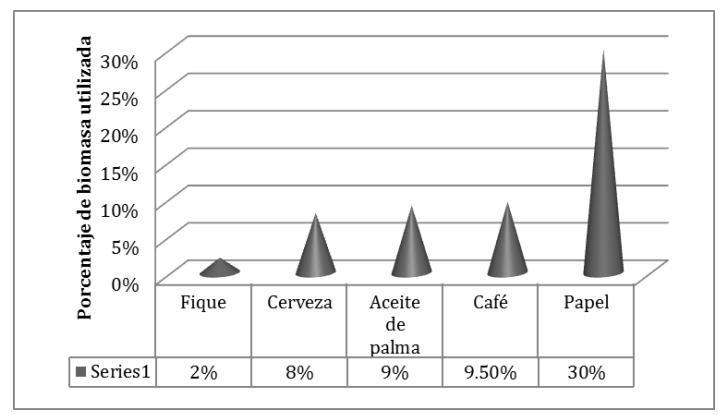

Figura 2 Aprovechamiento de los residuos industriales

\section{Metodología}

La realización de este estudio ha partido de la revisión de literatura científica para el proyecto de "Análisis y modelación de factores que inciden en el desempeño ambiental de empresas certificadas en ISO 14001. Caso de estudio: Área Metropolitana del Valle del Aburra", revisión que se ha realizado con base en datos de Redalyc, Dialnet, Scielo y Scopus considerando artículos escritos entre los años 2002 y 2017 en español, inglés y portugués teniendo en cuenta como algoritmos de búsqueda los términos ISO 14001, sistemas de gestión ambiental, certificación ambiental,

\section{Resultados}

En tabla 1 se observa que un gran número de empresas del sector industrial con SGA están realizando un reaprovechamiento de los desechos industriales, que luego son reintroducidos al ciclo, por ejemplo la industria de alimentos cuyos desechos vienen siendo transformados en nuevos subproductos para generación de energía y fertilizantes.

Tabla 1 Aprovechamiento de desechos por sectores industriales

\begin{tabular}{|l|l|l|}
\hline \multicolumn{1}{|c|}{ Industria } & \multicolumn{1}{|c|}{$\begin{array}{c}\text { Desecho } \\
\text { agroindustrial }\end{array}$} & Aprovechamiento \\
\hline Láctea & Suero & $\begin{array}{l}\text { Sustrato para la } \\
\text { producción de } \\
\text { ácido cítrico }\end{array}$ \\
\hline Azucarera & Bagazo & Bioetanol \\
\hline Maderera & Biomasa & Combustibles \\
\hline Aceites & Aceites Usados & Limpiadores \\
\hline $\begin{array}{l}\text { Ganadería } \\
\text { estabulada }\end{array}$ & Estiércol & Gas, Energía \\
\hline Plásticos & $\begin{array}{l}\text { Plástico y tetra- } \\
\text { brick }\end{array}$ & $\begin{array}{l}\text { Procesos } \\
\text { combustión de }\end{array}$ \\
\hline Papelera & Lodos & Combustión \\
\hline Aceites & Orujillo & $\begin{array}{l}\text { Pellets } \\
\text { calientes) }\end{array}$ \\
\hline $\begin{array}{l}\text { Telas } \\
\text { asfálticas }\end{array}$ & $\begin{array}{l}\text { Rollos de telas } \\
\text { asfálticas } \\
\text { defectuosos }\end{array}$ & Gases \\
\hline Curtiembre & Carnaza & Juguetes caninos \\
\hline Concretos & Residuos inertes & Concreto \\
\hline
\end{tabular}

Fuera de la certificación ISO 14001, las empresas están optando por obtener nuevas 
90

certificaciones, tales como la Rainforest Alliance, la FSC y la Carbón Trust Standard, como se ilustra en la tabla 2.

Tabla 2 Certificaciones ambientales

\begin{tabular}{cl}
\hline Certificación & \multicolumn{1}{c}{ Objetivo } \\
\hline Rainforest & El sello Rainforest Alliance \\
Alliance & Certified asegura a los \\
Certified & consumidores que el producto que \\
& están comprando ha sido \\
& cultivado y cosechado usando \\
& prácticas ambiental y socialmente \\
& responsable (Rainforest Alliance, \\
& 2012) \\
FSC (Forest & Se asegura que las empresas que \\
Stewardship & están a lo largo de la cadena de \\
Council) & suministro de la madera también \\
& cumplan con prácticas \\
& medioambientales (Forest \\
& Stewardship Council, 2012) \\
Es la certificación independiente \\
Standard & líder del mundo del impacto de \\
& una organización en los 'tres \\
& pilares' de la sostenibilidad \\
& ambiental: uso energético y \\
& emisiones de gas invernadero \\
& (CO2e), uso de agua yeneración \\
& de desechos. \\
\hline &
\end{tabular}

En la tabla 3 podemos observar como algunas empresas establecidas en Colombia aprovechan sus desechos.

Tabla 3 Empresas establecidas en Colombia que aprovechan los desechos industriales

\begin{tabular}{ll}
\hline \multicolumn{1}{c}{ Empresa } & $\begin{array}{l}\text { Aprovechamiento de } \\
\text { desechos }\end{array}$ \\
\hline Socya & $\begin{array}{l}\text { Recuperación de envases } \\
\text { PET }\end{array}$ \\
Cooperativa & Recicladores \\
Recimed & \\
Cementos argos & Sacos verdes \\
Natura cosméticos & $\begin{array}{l}\text { Alianza con Asociación de } \\
\text { recicladores }\end{array}$ \\
Enka & $\begin{array}{l}\text { Reciclaje botellas pet } \\
\text { Ricoh }\end{array}$ \\
& $\begin{array}{l}\text { Fotocopiadoras } \\
\text { reacondicionadas }\end{array}$ \\
\hline
\end{tabular}

\section{Conclusiones}

Cabe resaltar que un alto porcentaje de empresas que adoptan sistemas de gestión certificados, están presentando por fin un avance en la parte ambiental, y comienzan a darse cuenta que ellos tienen un valor agregado que favorece a la organización en aspectos tanto económicos, sociales, de imagen y ambientales.

Los desechos industriales deben ser considerados como productos y corresponde a la organización volverlos a reintroducir en los ciclos productivos sin estar sometidos a las limitaciones administrativas y técnicas asociadas a la condición de residuo. Sin embargo, las industrias les convienen ir un paso más allá de la norma ambiental, de la certificación, deben enfocarse en determinar las oportunidades que pueden representar todas las salidas que se generan de la transformación de la materia prima con la cual obtienen sus productos o servicios.

\section{Agradecimientos}

Este trabajo preliminar se enmarca en la tesis doctoral del primer autor en el programa de Doctorado en Ingeniería Ambiental de la Universidad de Antioquia. Los autores agradecen a la Profesora Silvia Teresa Morales Gualdrón por sus aportes a este trabajo en lo concerniente al área administrativa y organizacional.

\section{Referencias Bibliogràficas}

Agrocadenas Ministerio de Agricultura y desarrollo rural. (2006). Agroindustria y competitividad . Estructura y dinamica en Colombia. Bogota, Colombia. 
Cury R, K., Aguas M, Y., Martinez M, A., \& et al. (2017). Residuos Agroindustriales su impacto, manejo y aprovechamiento. Revista Colombiana de ciencia Anima(9), 122-132.

Escobar Cardenas, S. (2009). Realidad de los sistemas de gestion ambiental. Sotavento, 68-79.

Escobar Cardenas, S. (6 de Junio de 2009). Realidad de los sitemas de gestion ambienta. Sotavento MBA, 68-78.

Evia, G., \& Gudynas, E. (Agosoto de 2000). Valor agregado ambiental: Las nuevas relaciones entre ecologia y agropecuaria. Plan agropecuario(92), 52-56.

Forest Stewardship Council. (2012). Estandar Internacional FSC. FSC PRINCIPLES AND CRITERIA FOR FOREST STEWARDSHIP (Vol. $5)$.

Galarza, C. (1 de Octubre de 2012). Sustentator. Recuperado el 25 de Marzo de 2018, de http://www.sustentator.com/bloges/2012/10/certificacion-ambientaloportunidad-de-negocios-sustentables/

Pinzon Bedoya, M., \& Cardona Tamayo, A. (2008). Caracterizacion de la cascara de naranja para su uso como material bioadsorvente. Bistua, 6(1), 1-23.

Rainforest Alliance. (2012). Manual de Certificacion de Rainforest Alliance. Agricultura Sostenible. RA-Cert.

Rey, C. (2008). Sistema de Gestion Ambiental. Master en Ingenieria y gestion Medio ambiental, 7. EOI.

Singh, M., Brueckner, M., \& Kumar, P. (2015). Environmental management system ISO 14001: effective waste minimisation in small and medium enterprises in India. Journal of Cleaner Production(102), 285-301.

Tamayo Orbegozo, U., Vicente Molina, M., \& Izaguirre Olaizola, J. (24 de Abril de 2012). La gestion de residuos en la empresa: motivaciones para su implantacion y mejoras asociadas. Investigaciones Europeas de Direccion $y$ economia de la empresa., 216-277.

J. A. Vera Solano Ingeniero Ambiental Universidad de Pamplona - Especialista en Prevención y atención de desastres naturales del Tecnológico de Antioquia I.U - Magister en Ingeniería Ambiental de la Universidad de Pamplona. Estudiante de Doctorado en Ingeniería Ambiental Universidad de Antioquia, Docente tiempo completo ocasional programa de Ingeniería Ambiental Universidad de Pamplona. https://orcid.org/0000-0002-3415-146X

Julio Eduardo Cañón Barriga. Ingeniero civil Universidad Nacional de Colombia -Magister en Recursos Hidráulicos Universidad Nacional de Colombia. Doctorado Hydrology and Water Resources. The University of Arizona Department of Hydrology and Water Resources. Docente investigador Universidad de Antioquia.

*Para citar este artículo: Vera Solano J.A.; Cañón Barriga J.E.The added value of an environmental management system beyond certification.Universidad de Antioquia. Revista Bistua.2018.16(1):86-91.
+ Autor para el envió de correspondencia y la solicitud de las separatas Cañon Barriga J.E. .Universidad de Antioquia. Grupo de investigación: GAIA. julio.canon@udea.edu.co

Recibido: Diciembre 18 de 2016

Aceptado:Enero 20 de 2018 